\title{
Intervenciones basadas en Mindfulness en el tratamiento de adicciones a sustancias psicotrópicas
}

Inteventions based on Mindfulness in the Treatment of addictions to Psychotropic Substances

Intervenções baseada na Mindfulness no tratamento de dependencias em substâncias psicotrópicas

Diego Rodas Flores

ismael.rodasf@gmail.com

https://orcid.org/0000-0001-7827-4595

Posgrados Universidad Católica de Cuenca,
Cuenca-Ecuador
Geovanny Reivan Ortiz

greivano@ucacue.edu.ec https://orcid.org/0000-0003-0643-8022

Posgrados Universidad Católica de Cuenca, Cuenca-Ecuador
El consumo problemático de sustancias psicotrópicas incrementa año tras año; por tanto, el interés para desarrollar tratamientos eficaces para intervenir sobre estos trastornos lo hace en igual medida; en este contexto, una de la áreas de interés se centra en Intervenciones Basadas en Mindfulness (MBI), por lo cual, el presente artículo se plantea como objetivo general presentar la evidencia disponible sobre la eficacia que muestran las $\mathrm{MBI}$ para el tratamiento de las adicciones al alcohol, el cannabis y la cocaína. Se desarrolló una revisión sistemática en la que se encontraron 13 ensayos clínicos aleatorizados sobre la temática. Los resultados mostraron que las $\mathrm{MBI}$ son eficaces para promover la remisión de la conducta adictiva; así como para reducir la sintomatología depresiva y ansiosa. Sin embargo, en el presente artículo se discute sobre la dificultad para generalizar estos resultados y se insta a que investigaciones futuras se fundamente en una metodología sólida.

Palabras clave: IIntervenciones basadas en Mindfulness; adicciones; trastorno por consumo de alcohol; trastorno por consumo de cannabis; trastorno por consumo de cocaína
ABSTRACT

Problematic use (addiction) of psychotropic substances increases year after year; therefore, the interest to develop effective treatments to intervene on these disorders does so to the same extent; in this context, one of the areas of interest is focused on Mindfulness-Based Interventions (MBI), therefore, the present article aims to present the available evidence on the effectiveness of MBIs for the treatment of addictions. to alcohol, cannabis and cocaine. A systematic review was found in which 13 randomized clinical trials were found in which the application of $\mathrm{MBI}$, in an adult population over 18 years of age, was compared with other interventions. The results shown that MBIs are effective in promoting the remission of addictive behavior, as well as in preventing relapses, since they facilitate emotional regulation and the management of craving, in addition, their implementation has positive effects on the reduction of depressive and anxious symptoms. However, this article discusses the difficulty of generalizing these results and urges that future research be based on a solid methodology.

Key words: Mindfulness-based interventions; Addictions; alcohol use disorder; alcohol use disorder; cannabis use; cannabis use disorder; cocaine use disorder
RESUMO

O uso problemático de substâncias psicotrópicas aumenta ano após ano; portanto, o interesse em desenvolver tratamentos eficazes para intervir nesses distúrbios o faz na mesma medida; Nesse contexto, uma das áreas de interesse centra-se nas Intervenções Baseadas em Mindfulness (MBI), portanto, este artigo tem como objetivo apresentar as evidências disponíveis sobre a eficácia dos MBIs no tratamento de dependências ao álcool, cannabis e cocaína. Foi desenvolvida uma revisão sistemática na qual foram encontrados 13 ensaios clínicos randomizados sobre o assunto. Os resultados mostraram que os MBIs são eficazes na promoção da remissão do comportamento aditivo; bem como para reduzir os sintomas depressivos e ansiosos. No entanto, este artigo discute a dificuldade de generalizar esses resultados e preconiza que pesquisas futuras sejam baseadas em uma metodologia sólida.

Palavras-chave: Intervenções baseadas no cuidado;

vícios; distúrbios relacionados com o uso de álcool; desordem de uso de cannabis; desordem de uso da cocaína 


\section{INTRODUCCIÓN}

Las Intervenciones Basadas en Mindfulness (MBI) son tratamientos psicológicos que forman parte de la tercera ola de terapias conductuales, y que fundamentan sus prácticas en el entrenamiento de la consciencia plena (mindfulness); la cual, emerge al focalizar la atención en el momento presente de forma intencional, manteniendo una actitud de aceptación, se trata pues de una capacidad psicológica relativamente estable, sin embargo, la práctica meditativa constante facilita su adiestramiento (Hervás et al., 2016).

Ursa (2018) narra que la meditación aplicada en los ejercicios de Mindfulness proviene del budismo; se refieren a esta como un conocimiento ancestral llevado a la práctica que procura la aceptación del pensamiento, la toma de conciencia sobre las experiencias, a partir de lo cual, facilita el distanciamiento de las conductas desadaptativas y de los pensamientos distorsionados.

Por lo mencionado con antelación, Jon Kabat - Zinn, en 1979, desarrolló el programa Reducción del Estrés basado en Mindfulness (MBSR), en base a la meditación budista, para el tratamiento de personas diagnosticadas con enfermedades crónicas (Black, 2014). A partir de este primer protocolo, se formularon de manera posterior otros, que se aplican en diferentes psicopatologías, por ejemplo, en depresión, manía, ansiedad, psicosis y consumo de sustancias (Hervás et al., 2016 y Korecki et al., 2020).

Pese a la eficacia que muestran estás intervenciones aplicadas en el tratamiento de las adicciones (Li et al., 2017; y, Korecki et al., 2020), Grant et al. (2017), refieren que, no existe evidencia concluyente para sugerir su aplicación, pues su campo de estudio es reducido; además de que, los resultados positivos que se extraen de los Ensayos Clínicos Aleatorizados (ECA), debido a la cantidad de la muestra o a la ausencia de información en los informes, no permiten generalizaciones.

Sumada a esta dificultad se encontró que la ingesta problemática de sustancias psicoactivas aumenta cada año (Bautista et al., 2019). De hecho, de acuerdo con la Oficina de las Naciones Unidas contra la Droga y el Delito (UNODC, 2019), entre 2016 y 2019, la prevalencia a nivel mundial del Trastorno por Consumo de Sustancias (TCS) incrementó en un $15 \%$, para la fecha se estimó que 35 millones de individuos fueron diagnosticados con esta psicopatología; es decir, el $0,71 \%$ de personas cuya edad se encuentra entre $15 \mathrm{y}$ 64 años; siendo la población de entre 18 y 25 años, quienes presentan los valores máximos en la ingesta de sustancias psicotrópicas.

En este sentido, las drogas que se consumen con mayor prevalencia a nivel mundial son: alcohol (283 millones de consumidores), cannabis (192 millones) y cocaína (19 millones) (UNOCD, 2019 y Organización de Estados Americanos, 2019). Por otra parte, se reporta que apenas una de cada siete persona que presentan adicción reciben un tratamiento; y que, existe un elevado índice de recaídas, se considera que la mayoría de adultos que reciben terapia recaen antes de los 12 meses de remisión, y de abandono; sumado a ello, encontramos que la respuesta sanitaria existente es insuficiente e ineficaz, además de que no sustenta su aplicación en evidencia 
científica (Bautista et al., 2019; McLellan et al., 2000; y, UNODC, 2019).

En este contexto, debido a la escasa eficacia de los tratamientos actuales tanto para procurar la permanencia en el tratamiento como la remisión de la conducta adictiva, así como, al incremento en la tasa de consumo de sustancias psicotrópicas; Grant et al. (2017), instan a desarrollar investigaciones que se enfoquen en determinar la factibilidad de aplicar MBI en contextos de salud pública y privada, y la eficacia que muestran al aplicarse de manera combinada o en comparación con otras terapias, pues de esta manera, podrían constituirse como alternativas psicoterapéuticas viables.

Por tanto, la presente revisión sistemática, sobre las MBI para el tratamiento de las adicciones al alcohol, cocaína y cannabis en población adulta, podría resultar de interés para las personas que buscan recibir un tratamiento, sus familias, los profesionales, la academia y entidades de la salud involucrados en este campo.

\section{Trastorno por consumo de sustancias}

EITCS, o adicción, es una psicopatología que se caracteriza, de acuerdo con la Asociación Americana de Psiquiatría (APA, 2014), por la ingesta reiterada de sustancias psicoactivas que producen intoxicación; deseo apremiante de consumir; graves problemas para disminuir o abandonar de forma voluntaria el consumo (pese a las dificultades que este acarrea a nivel laboral, personal, social, familiar y académico); necesidad de hacerse con la sustancia a pesar de cualquier obstáculo que pudiera existir; $y$, si esto no es posible, síndrome de abstinencia.
Para diagnosticar el TCS, es necesario que se identifiquen, por lo menos, dos de los síntomas citados en el párrafo previo, mismos que deberán estar presentes de forma continuada durante un periodo mínimo de 12 meses; una vez realizado el diagnóstico, se debe especificar la gravedad (leve, moderada o grave) en función del número de criterios diagnósticos que se cumplan (APA, 2014).

Es importante, en este punto, realizar una aproximación conceptual sobre algunos términos asociados al TCS. Las drogas o sustancias psicoactivas son cualquier producto externoquealseringerido, inhalado o inyectado modifica el estado de conciencia, el ánimo, la cognición o la motricidad; y que, en función del cambio que genera en el Sistema Nervioso Central, se clasifican en depresoras (alcohol), estimulantes (cocaína) o alucinógenas (cannabis) (Sateler et al., 2019). Por otra parte, se entiende por abuso a la ingesta continuada de la droga pese a las dificultades que esto genera; mientras que, la dependencia es la administración reiterada de un estupefaciente que produce tolerancia; este último término se refiere a un estado de adaptación en el que se aminora la reacción del cuerpo ante la ingesta de la misma cantidad de sustancia o en el que se requiere mayor sustancia para alcanzar el efecto deseado (Becoña y Cortés, 2010).

Cuando la droga no es ingerida se produce el síndrome de abstinencia, que se caracteriza por la presencia de ansiedad y dificultades para dormir (APA, 2014). Mientras que, el estado en el que cese el consumo es prolongado, se conoce como remisión. Es importante considerar esto, pues de acuerdo con Bautista et al. (2019), los tratamientos actuales presentan una alta tasa 
de abandono y posterior recaída en el consumo de sustancias. Entendiendo la recaída como retomar la ingesta de la sustancia, después de haber cursado un periodo de remisión.

\section{Intervenciones basadas en Mindfulness y adicciones}

De acuerdo con Lerma et al. (2015), ante la ausencia de tratamientos eficaces para intervenir sobre la adicción, una de las aproximaciones psicoterapéuticas que mayor desarrollo ha tenido y éxitos ha conseguido en los últimos años son las MBI. En este contexto, sobre las $\mathrm{MBI}$ se han desarrollado investigaciones que han buscado identificar aspectos teóricos y metodológicos en los que basan sus prácticas. Es así como, en un ECA Bayir y Aylaz (2020), obtuvieron que, una vez finalizado el tratamiento, en el grupo experimental la media en la percepción de autoeficacia incrementó de 76,50 a 85,50 , lo que implicó una diferencia estadísticamente significativa $(p<0.05)$ con relación al grupo control; estos resultados les permitieron concluir que la eficacia de MBI radica en el incremento de la autoeficacia percibida.

Postura con la que concuerda Brewer et al. (2010), cuando señala, refiriéndose al modelo de regulación bottom up, que el Mindfulness, al estimular el desarrollo de la atención y la aceptación, permite detectar afectos negativos, aceptarlos y controlar la conducta. Esto fue corroborado por Witkiewitz et al.(2013), cuando al estudiar en 168 participantes los efectos que la MBRP tiene sobre la ansiedad por consumo postratamiento; encontraron que las variables interdependientes de aceptación, conciencia y no juicio mediaban significativamente en la relación existente entre recibir MBRP y reducir los niveles autoinformados de ansiedad.

Mientras que, en la investigación realizada por Witkiewitz y Villaroel (2009), en 774 participantes adultos, se identificó que existe una correlación positiva y estadísticamente significativa entre afecto negativo y consumo de alcohol; $y$, que las $\mathrm{MBI}$ reducen los afectos negativos, por tanto, el consumo de sustancias, teniendo un rango de efecto igual a $0,13-0,33$. Por otra parte, en una revisión documental realizada por Zgierska et al. (2009), identificaron que no existe un formato único para la aplicación de MBI en adicciones; sin embargo, los protocolos coinciden en que: satisfacer las expectativas del paciente, brindar el tratamiento ofrecido, y tener capacidad para seleccionar las mejores técnicas según las necesidades del cliente, son aspectos claves para alcanzar la remisión en el uso de la sustancia.

En este sentido, Skanavi et al. (2011), en una revisión sistemática, encontraron que la

eficacia de las MBI radica en que, debido a su base teórica Cognitivo Conductual, tienden a promover la práctica de habilidades; además, señalan que los protocolos incorporan terapias grupales, así como, ejercicios de atención plena que se ejecutan durante seis días a la semana, mínimamente por 45 minutos. Sancho et al. (2018), a partir de los resultados obtenidos en una revisión sistemática, acotan que el número indicado de sesiones oscila entre siete y $12, y$ el tiempo de cada una es de tres horas.

Finalmente, el interés también se ha centrado en identificar la eficacia que muestran estás terapias para mantener la remisión del consumo. Por cuanto, en un meta-análisis 
realizado sobre 42 investigaciones, $\mathrm{Li}$ et al. (2017) identificaron que el abuso de alcohol entre los adultos, que recibían $\mathrm{MBI}$, se reducía significativamentea los 3 meses deseguimiento; además que, estos son tratamientos positivos para aminorar el consumo de marihuana en población femenina.

En este contexto, en la revisión sistemática realizada por Cavicchioli et al (2020), a 30 estudios, en los que se incluyeron $\mathrm{MBI}$ para tratar el consumo de cocaína, cannabis y alcohol, determinaron que estas psicoterapias son tan efectivas como la aplicación de tratamientos basados en evidencia para la intervención sobre adicciones, pues reducen la cantidad y frecuencia de la ingesta de las drogas, así como problemas relacionados con el uso de sustancias y el deseo de consumir, por lo cual contribuye a mantener la remisión continuada (1 año) de la psicopatología; pese a ello, refieren los autores que las $\mathrm{MBI}$ no son empleadas como intervenciones de primera mano para el tratamiento de las adicciones.

Por ello, en el presente artículo se plantea como objetivo general presentar la evidencia disponible sobre la eficacia que muestran las $\mathrm{MBI}$ en el tratamiento de las adicciones al alcohol, el cannabis y la cocaína. Por otra parte, la hipótesis de partida es la siguiente: las MBI son eficaces para mantener la remisión del consumo hasta un año después de finalizado el tratamiento.

\section{MÉTODO}

\section{Protocolo}

La estructura y redacción de este reporte se fundamentó en el Manual Cochrane para Revisiones Sistemáticas (Centro Cochrane
Iberoamericano, 2011); así como, en la declaración de los Elementos de Referencia para Informes de Revisiones Sistemáticas y Metaanálisis 2009 (PRISMA), herramienta que fue diseñada para fomentar una presentación trasparente y completa del proceso ejecutado durante la revisión y de los resultados obtenidos (Moher et al., 2009). Además, se empleó el instrumento para la evaluación crítica de las revisiones sistemáticas de los estudios realizados sobre intervenciones sanitarias en su versión 2 (AMSTAR-2), que permite evaluar la calidad de las revisiones sistemáticas que se realizan sobre ECA y no aleatorizados (Ciapponi, 2018).

\section{Criterios de Inclusión}

Los criterios de inclusión empleados fueron: los ECA publicados en revistas científicas, en inglés o español, hasta junio de 2021. Así como, los estudios realizados en población adulta (> 18 años) que presente diagnóstico de Trastorno por Consumo de Alcohol, Cannabis o Cocaína establecido a partir del Manual Diagnóstico y Estadístico de Trastornos Mentales (APA, 2014) o de la Clasificación Internacional de Enfermedades (Organización Panamericana de la Salud, 2003); o, reclutados en un entorno clínico de tratamiento para adicciones. Además de las investigaciones que comparen la aplicación de una $\mathrm{MBI}$, sola o combinada, con otra intervención psicológica, psicoeducativa, farmacológica y/o grupo control. Aunado a los ensayos que proporcionen medidas fiables y validas de reporte pretratamiento, post tratamiento $\mathrm{y} / \mathrm{o}$ de seguimiento $\mathrm{y}$ por último, las investigaciones que reporten una descripción de las características de la $\mathrm{MBI}$ aplicada. 


\section{Criterios de Exclusión}

Los criterios de exclusión empleados fueron los ECA publicados en revistas científicas antes de junio de 2015; las investigaciones en las que al grupo experimental se les administró farmacoterapia y los estudios en los que se incluyeron personas menores de 18 años.

\section{Identificación de estudios}

Se realizó una búsqueda de literatura en las bases de datos Web of Science, Scopus, PubMed y ProQuest, en específico, en Psychology, utilizando las siguientes palabras clave: "mindfulness", "mindfulness intervention", "mindfulness treatment", "mindfulness-based relapse prevention", "mindfulness-oriented recovery enhancement", "moment-by-moment in women's recovery", "mindful awareness in body-oriented therapy", "mindfulness training for smokers", "acceptance and commitment therapy", "dialectical behavior therapy" " "mindfulness based intervention" en combinación con el nombre de cada una de las sustancias ("alcohol", "cannabis", "marijuana" o "cocaína").

La búsqueda se complementó con las referencias citadas en las revisiones sistemáticas y metaanálisis realizados por Bautista et al. (2019), Cavicchioli et al. (2018), Grant et al. (2017), Korecki et al. (2020) Li et al. (2017) y Sancho et al. (2018). La búsqueda se realizó entre el 25 y el 28 de junio de 2021.

\section{Selección de los estudios}

La selección de los artículos constó de dos momentos, que fueron: una revisión de los títulos y de los resúmenes para determinar que investigaciones deberían ser examinadas a texto completo; y, una revisión de los artículos que pasaron el primer filtro, ambas, en función de los criterios de inclusión y exclusión mencionados con antelación. Cuando existió incertidumbre respecto a la integración de un artículo, se solicitó al coautor la revisión de este y la valoración sobre la pertinencia de su inclusión.

\section{Extracción de datos}

Para la extracción de los datos se utilizó una tabla que permitió obtener: nombres de los autores de cada artículo; momentos y herramientas empleados para la recolección de datos; tamaño y características de la muestra; descripción de las MBI y de las intervenciones aplicadas en el grupo experimental y control, respectivamente; y resultados obtenidos.

\section{Evaluación de la calidad metodológica de los estudios}

Los ECA fueron evaluados utilizando la Lista de verificación de la metodología para Ensayos Clínicos Aleatorizados del Instituto Nacional de Salud y Excelencia Clínica (NICE), esta herramienta fue diseñada para determinar la validez interna de los estudios y valorar el riesgo de sesgo; consta de cuatro secciones, cada una se enfoca en un sesgo específico, a saber: selección, formalidad, deserción y detección (Arnold y Bautmans, 2014). Para cada uno de los ítems contenidos en las secciones se proporcionaron una de las siguientes respuestas "si", "no", "no está claro" y "no aplica".

Por otra parte, también se empleó la Escala de evaluación de la metodología de estudios de los resultados en psicoterapia (POMFR), que 
consta de 22 ítems y permite evaluar el rigor metodológico de las intervenciones; cada ítem se puntúa de cero a dos, donde cero significa pobre, uno justo y dos bueno; los estudios pueden alcanzar un máximo de 44 puntos, interpretándose que a mayor puntaje existe mayor rigor metodológico (Öst, 2008).

\section{DESARROLLO Y DISCUSIÓN}

\section{Selección de estudios}

La búsqueda en las bases de datos generó un total de 389 resultados, a los cuales se adicionaron seis artículos identificados en las

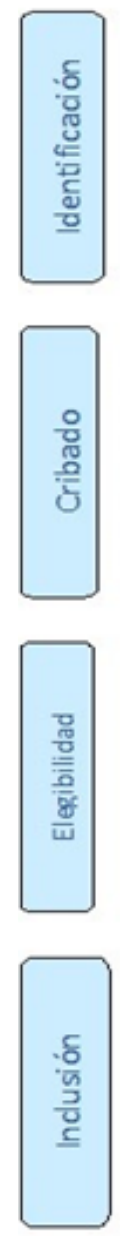

$$
(n=389)
$$

referencias citadas por Bautista et al. (2019), Cavicchioli et al. (2018), Grant et al. (2017), Korecki et al. (2020) Li et al. (2017) y Sancho et al. (2018). Del total $(n=395)$, se eliminaron 124 artículos duplicados; después de revisar los títulos y los resúmenes se excluyeron 219 artículos más; y, 39 luego de la lectura a texto completo (ver Figura 1); por tanto, 13 fueron incluidos en la revisión sistemática.
Reg istros adicionales encontra dos en otros artículos ( $n=6)$

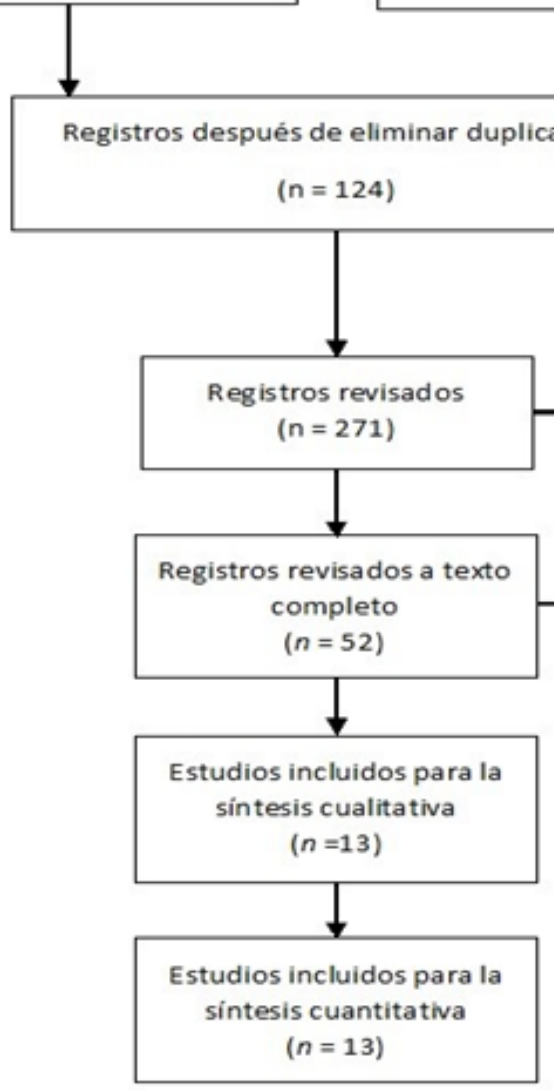

Registros excluidos $(n=219)$

Figura 1. Diagrama de flujo de la revisión sistemática y el proceso de selección. Adaptado de "Emotion regulation as a transdiagnostic treatment construct across anxiety, depression, substance, eating and borderline personality disorders: A systematic review". Por Sloan et. Al.; (2014). Clinical Phychology Review, 57(1), p. 145. 


\section{Resultados de los estudios incluidos}

En la Tabla 1 se ofrece una descripción general de los estudios $(n=13)$ incluidos en la presente revisión sistemática en función del autor principal, el país donde se realizó el estudio, las características de la muestra y de la intervención; así como, las herramientas que se emplearon para identificar los resultados. El número total de participantes fue 1272 adultos; de los cuales, 707 fueron mujeres y 565 hombres.

\section{Evaluación de la calidad metodológica}

Para evaluar la calidad metodológica de las intervenciones se empleó el POMRF. De 44 puntos posibles, los resultados obtenidos en los estudios incluidos se ubicaron entre 15 y 30 (Tabla 1), con una media de 21.85 y una desviación estándar de 5.28; lo que sugiere una variabilidad significativa en torno al rigor metodológico con que se implementaron los tratamientos en las investigaciones.

Para profundizar en la comparación sobre la calidad metodológica de los tratamientos se adaptó el método de comparación empleado porSwain, etal.,(2013)yseconsidera como “muy por debajo" a los estudios que se encuentran a más de una desviación estándar por debajo de 21.85 ( $n=3$ ); como "por debajo" a los que se encuentran una desviación estándar debajo de la media $(n=3)$, como "por encima" a los que se encuentran una desviación estándar sobre la media $(n=5)$; y, como "muy por encima" a los que se encuentran a más de una deviación estándar sobre la media $(n=2)$.

\section{Diseño de los estudios}

Todos los estudios incluidos fueron ECA $(n=13)$. De estos, tres recibieron la máxima calificación, "bueno”, por comparar un MBI con dos tratamientos activos $(n=1)$, o una MBI con un tratamiento activo bien detallado $(n=2)$; mientras que, cuatro recibieron la calificación de "justo", pues compararon el tratamiento experimental con un tratamiento habitual descrito de manera adecuada; finalmente, seis recibieron la calificación de "pobre" pues se comparó una $\mathrm{MBI}$, y se proporcionó una descripción vaga del tratamiento habitual ( $n=$ 3) o no se describió el tratamiento habitual ( $n$ $=3)$.

\section{Terapeutas}

De los 13 artículos revisados, en nueve participaron al menos dos terapeutas en el desarrollo de las intervenciones; sin embargo, en ninguno se evaluó la influencia que tuvo el terapeuta sobre los resultados. Por otra parte, en relación con la experiencia que tenían los terapeutas, en nueve de los 13 artículos se indicó que se trataban de profesionales entrenados tanto en $\mathrm{MBI}$ como en el abordaje del TCS.

\section{Adherencia y Competencia}

Respecto a la adherencia al tratamiento, en ocho de los artículos incluidos fue evaluada de manera frecuente mediante el empleo de escalas detalladas. 


\section{RESULTADOS Y DISCUSIÓN}

En la Tabla 1 se presenta una breve descripción de los resultados identificados en torno al consumo de sustancias. Debido a la heterogeneidad de los estudios, el tamaño del efecto de las intervenciones se muestra en diferentes formatos (por ejemplo: $r, \eta 2$, g y d).

En 12 de las investigaciones revisadas se llevó a cabo un análisis longitudinal de la influencia que tuvo la MBI sobre algún síntoma clínico (ansiedad, depresión, etc.) o sobre el consumo de sustancias.

Intervenciones Basadas en Mindfulness y práctica de Mindfulness

La MBRP fue la MBI que con mayor frecuencia se aplicó en el grupo experimental $(n=7)$, pese a que el formato de su aplicación fue heterogéneo, existen algunos puntos en común, los siguientes: en todas las intervenciones fue empleada como un proceso de tratamiento grupal $(n=7)$ en el que se abordaron ejercicios de meditación guiada y de prevención de recaídas, así como, revisión de tareas para casa; $y$, en seis se aplicó en ocho sesiones, mismas que oscilaron entre los 75 y 120 minutos. Además, en dos se aplicó MBRP en el formato sugerido por Bowen et al., (2011); mientras que, en otras dos se adaptó este formato para que respondiera a las necesidades específicas de las personas con TCA.

Además, en tres investigaciones, que analizaron la adherencia al tratamiento, se identificó que la tasa de asistencia y permanencia es mayor en las MBI que en las intervenciones que se ofrecieron al grupo control. Por otra parte, en seis artículos se identificó que, al finalizar el tratamiento los participantes que recibieron una $\mathrm{MBI}$ desarrollaron y practicaron Mindfulness con mayor frecuencia que los participantes del grupo control; sin embargo, en tres estudios reportan que esta diferencia no se mantuvo en el seguimiento posterior.

\section{Síntomas clínicos}

De los 13 artículos revisados, en seis se examinó el impacto que tuvo la MBI sobre la disminución de la sintomatología depresiva al finalizar el tratamiento y en el seguimiento posterior, en cuatro se identificó una reducción de los síntomas y una mayor eficacia en comparación con el grupo control, mientras que, en dos no existió diferencia entre los grupos.

Otro de los síntomas clínicos analizados fue la ansiedad, los resultados de dos investigaciones sugieren que las $\mathrm{MBI}$ son adecuadas para intervenir sobre la sintomatología ansiosa, pues generan efectos positivos que son superiores a los del grupo control. Por otra parte, en cuatro investigaciones se reportó una disminución del estrés al finalizar el tratamiento, mientras que en una se mencionó un incremento de la capacidad para tolerarlo.

\section{Efectos sobre el consumo de sustancias}

Respecto al craving, se identificó que las $\mathrm{MBI}$ son eficaces para disminuir el deseo de consumo al finalizar el tratamiento $(n=7)$, en el seguimiento de hasta tres meses $(n=5)$, de 
hasta seis meses $(n=2)$ y de hasta 12 meses $(n$ $=1$ ). Por cuanto, se ubica a estas intervenciones como eficaces para prevenir las recaídas en el consumo de sustancias.

\section{Efectos sobre el consumo de alcohol}

Las MBI aplicadas mostraron ser eficaces para disminuir la cantidad de bebidas alcohólicas ingeridas por día (Price et al., 2019; Witkiewitz et al., 2019 y Zgierska., 2019), para promover la remisión de la conducta adictiva al finalizar el tratamiento (Wongtongkam et al., 2017), y mantener el estado de abstinencia en el seguimiento a los dos meses (Witkiewitz et al., 2019), a los seis meses (Davis et al., 2018) y a los 12 meses (Price et al., 2019). Además, se asoció la práctica de mindfulness con la reducción del consumo de alcohol y se identificó que, mientras más sesiones de MBI se reciben y más practica de meditación se realiza, la cantidad y frecuencia de la ingesta de la sustancia tiende a reducirse (Witkiewitz et al., 2019 y Zgierska et al., 2019).

Por otra parte, en una investigación en la que se combinó MBRP con Estimulación Magnética Transcraneal se identificó que, tanto al finalizar el tratamiento como en el seguimiento posterior, los pacientes redujeron su respuesta cerebral ante la exposición a señales de alcohol, lo que funcionaría como un factor de protección ante posibles recaídas (Brown et al., 2020).

Es importante indicar que para el desarrollo de este apartado se incluyeron cuatro artículos que abordaron de manera exclusiva el TCA; $y$, otros dos en los que se abordó está patología junto con el consumo de otras sustancias.

\section{Efectos sobre el consumo de cocaína}

Endosartículos se proporcionó información sobre la remisión del consumo de cocaína. En uno de los estudios no se identificaron diferencias significativas al comparar la MBRP con la Educación en Salud; sin embargo, cuando los pacientes del grupo experimental presentaban de manera comórbida a la adicción, depresión o ansiedad, fueron menos propensos a ingerir cocaína que los del grupo control (Glasner et al., 2016).

En el otro estudio se reportó un incremento en el porcentaje de días en que los participantes, que recibieron MBRP, consumían cocaína al finalizar el tratamiento, pero en el seguimiento a los tres meses existió una disminución, de aproximadamente la mitad en comparación con los datos recogidos antes de la intervención (Machado et al., 2020).

\section{Efectos sobre el consumo de cannabis}

En dos artículos se reportó población que presentó consumo de cannabis. En uno de los estudios se señaló un decremento de la ingesta de la droga tanto al finalizar la intervención como en el seguimiento posterior, sin embargo, al compararlo con TAU no existieron diferencias estadísticamente significativas (Machado et al., 2020). Mientras que, en el otro estudio se identificó que los participantes que recibieron MABT mostraron más días en abstinencia que los del grupo control tanto al finalizar el tratamiento, como en el seguimiento de hasta seis y 12 meses (Price et al., 2019). 
Tabla 1. Resultados de los estudios incluidos.

\begin{tabular}{|c|c|c|c|c|c|c|c|c|}
\hline \multirow[b]{2}{*}{ Autores } & \multicolumn{4}{|c|}{ Característica de la muestra } & \multicolumn{2}{|l|}{ Tratamiento } & \multicolumn{2}{|l|}{ Resultados } \\
\hline & País & $\begin{array}{l}\text { Diagnóstico } \\
\text { droga consumida }\end{array}$ & Sexo & Tamaño & Sesiones & Herramientas & Consumo de sustancias & POMFR \\
\hline \multirow[t]{3}{*}{$\begin{array}{l}\text { Glasner } \\
\text { et al., } 2016\end{array}$} & \multirow[t]{3}{*}{ USA } & \multirow[t]{3}{*}{$\begin{array}{l}\text { StD / } 55,6 \% \\
\text { cocaína }\end{array}$} & \multirow[t]{3}{*}{$\begin{array}{l}28,6 \% \text { mujeres } \\
71.4 \% \text { hombres }\end{array}$} & \multirow[t]{3}{*}{63} & $\begin{array}{l}\text { CM + MBRP: } 2 \text { sesiones de } 75 \\
\text { minutos, durante } 4 \text { semanas, } \\
8 \text { sesiones en total. }\end{array}$ & \multirow[t]{3}{*}{ UTS; ASI } & $\begin{array}{l}\text { Orina libre de estimulantes, durante } \\
\text { el tratamiento, no fue diferente } \\
\text { entre los grupos (MBRP }=0.73^{*} \text { y HE }\end{array}$ & \multirow[t]{3}{*}{22} \\
\hline & & & & & $\begin{array}{l}\text { CM + HE: } 2 \text { sesiones de } 75 \\
\text { minutos, durante } 4 \text { semanas, }\end{array}$ & & $\begin{array}{l}\left.=0.70{ }^{\star}\right) ; \text { tampoco en el seguimiento } \\
(\mathrm{OR}>1) .\end{array}$ & \\
\hline & & & & & 8 sesiones en total. & & $\begin{array}{l}\text { Los participantes que recibieron } \\
\text { MBRP, mostraron grandes beneficios } \\
\text { en la probabilidad de reducir el } \\
\text { uso de estimulantes }\left(\mathrm{OR}=0.78^{\star}\right) \text {, } \\
\text { resultados similares se encontraron } \\
\text { en el grupo GAD (OR }=68^{\star} \text { ) en } \\
\text { comparación con el grupo HE. }\end{array}$ & \\
\hline \multirow[t]{2}{*}{$\begin{array}{l}\text { Valls } \\
\text { et al., } 2016\end{array}$} & \multirow[t]{2}{*}{ España } & \multirow[t]{2}{*}{$\begin{array}{l}\text { PSUD / alcohol } \\
\text { y cocaína }\end{array}$} & \multirow[t]{2}{*}{$\begin{array}{l}\text { Mujeres = } 11 \\
\text { Hombres }=21\end{array}$} & \multirow[t]{2}{*}{32} & $\begin{array}{l}\text { GMT + MM: } 8 \text { sesiones de } \\
120 \text { minutos de GMT y } 8 \\
\text { sesiones de } 40 \text { minutos de } \\
\text { MM, durante } 8 \text { semanas. }\end{array}$ & \multirow[t]{2}{*}{$\mathrm{NI}$} & $\mathrm{NI}$ & \multirow[t]{2}{*}{15} \\
\hline & & & & & TAU: NI & & & \\
\hline $\begin{array}{l}\text { Zemestani } \\
\text { y Ottaviani, } \\
2016\end{array}$ & Irán & $\begin{array}{l}\text { SUD / } 17.8 \% \\
\text { Cocaína } \\
\text { and } 13.7 \% \\
\text { Marihuana }\end{array}$ & $\begin{array}{l}\text { 20.3\% Mujeres } \\
\text { 79.7\% Hombres }\end{array}$ & 74 & $\begin{array}{l}\text { MBRP: } 3 \text { grupos separados } \\
\text { recibieron } 8 \text { sesiones de } 2 \\
\text { horas, durante } 8 \text { semanas. } \\
\text { TAU: } 3 \text { grupos separados } \\
\text { recibieron } 8 \text { sesiones de } 2 \\
\text { horas, durante } 8 \text { semanas. }\end{array}$ & PACS & $\begin{array}{l}\text { El grupo MBRP obtuvo un puntaje } \\
\text { menor que } 20 \text { en craving en el } \\
\text { post tratamiento }(m=12.0) \text { y en } \\
\text { el seguimiento ( } m=11.8) \text {. MBRP } \\
\text { mostró mejores resultados para } \\
\text { prevenir recaídas que TAU. }\end{array}$ & 23 \\
\hline
\end{tabular}




\begin{tabular}{|c|c|c|c|c|c|c|c|c|}
\hline \multirow[b]{2}{*}{ Autores } & \multirow[b]{2}{*}{ País } & \multicolumn{3}{|c|}{ Característica de la muestra } & \multicolumn{2}{|l|}{ Tratamiento } & \multicolumn{2}{|l|}{ Resultados } \\
\hline & & $\begin{array}{c}\text { Diagnóstico } \\
\text { droga consumida }\end{array}$ & Sexo & Tamaño & Sesiones & Herramientas & Consumo de sustancias & POMFR \\
\hline $\begin{array}{c}\text { Shorey } \\
\text { et al., } 2017\end{array}$ & USA & $\begin{array}{c}\text { SUD / } \\
61.5 \% \text { Alcohol, } \\
1.8 \% \text { Cocaína } \\
\text { y } 0.9 \% \text { Cannabis }\end{array}$ & $\begin{array}{l}30 \text { mujeres } \\
87 \text { hombres }\end{array}$ & 117 & $\begin{array}{l}\text { ACT + TAU: } 2 \text { sesiones de } \\
1.5 \text { horas por semana, en } \\
\text { total } 8 \text {. Las intervenciones } \\
\text { incluyeron ejercicios de: } \\
\text { MBRP, MBSR and ACT. } \\
\text { TAU: } 1 \text { sesión por día, } \\
\text { durante } 28 \text { días, basada en } \\
\text { el modelo de } 12 \text { pasos. }\end{array}$ & PACS & $\begin{array}{l}\text { La diferencia en el tamaño del } \\
\text { efecto entre grupo fue pequeña, } \\
\text { ACT reportó menores síntomas } \\
\text { de craving }(d=0.23) \text { y una mayor } \\
\text { flexibilidad psicológica respecto al } \\
\text { uso de sustancias }(d=0.20) \text {. }\end{array}$ & 19 \\
\hline $\begin{array}{l}\text { Wongtongkam } \\
\text { et al., } 2017\end{array}$ & Tailandia & $A D$ & $\begin{array}{c}0 \text { mujeres } \\
55 \text { hombres }\end{array}$ & 55 & $\begin{array}{l}\text { VM: } 2 \text { horas de VM durante } \\
5 \text { días. } \\
\text { TAU: } \mathrm{NI}\end{array}$ & DDQ-R & $\begin{array}{l}\text { Ambos grupos abandonaron el consumo } \\
\text { de cigarrillos y de alcohol (datos no } \\
\text { rexportados). }\end{array}$ & 16 \\
\hline $\begin{array}{l}\text { Zgierska } \\
\text { et al., } 2017\end{array}$ & USA & $A D$ & $\begin{array}{l}43.1 \% \text { mujeres } \\
56,9 \% \text { hombres }\end{array}$ & 123 & $\begin{array}{l}\text { MBRP-A + TAU: } 8 \text { sesiones } \\
\text { grupales de } 2 \text { horas. } \\
\text { TAU: NI }\end{array}$ & $\begin{array}{l}\text { Concentración } \\
\text { de alcohol en } \\
\text { la sangre }\end{array}$ & $\begin{array}{l}\text { En la pre intervención los grupos } \\
\text { no mostraron diferencias respecto } \\
\text { al consumo de alcohol ( } p=0.406- \\
0.916 \text { ) y a sus consecuencias ( } p= \\
0.807 \text { ). No refieren resultados de la } \\
\text { post intervención y del seguimiento. }\end{array}$ & 19 \\
\hline $\begin{array}{c}\text { Davis } \\
\text { et al., } 2018\end{array}$ & USA & $\begin{array}{l}\text { SUD / Alcohol } \\
\text { y otras drogas }\end{array}$ & $\begin{array}{l}35 \% \text { mujeres } \\
65 \% \text { hombres }\end{array}$ & 79 & $\begin{array}{l}\text { TAU + MBRP: TAU + } 2 \\
\text { sesiones de } 1.5 \text { horas de } \\
\text { MBRP, durante } 4 \text { semanas. } \\
\text { TAU: } 2 \text { sesiones de } 1.5 \\
\text { horas de } 12 \text { pasos }+ \\
\text { sesiones grupales de CBT, } \\
\text { durante } 4 \text { semanas. }\end{array}$ & $\begin{array}{l}\text { SFS; Craving } \\
\text { Scale from } \\
\text { GAIN; } \\
\text { Assessment } \\
\text { instrument. }\end{array}$ & $\begin{array}{l}\text { La intervención de MBRP generó un } \\
\text { menor uso de sustancias ( } \mathrm{d}=-0.58) \\
\text { y de craving }(\mathrm{d}=-0.77) \text { en el post } \\
\text { tratamiento y en el seguimiento. }\end{array}$ & 30 \\
\hline
\end{tabular}




\begin{tabular}{|c|c|c|c|c|c|c|c|c|}
\hline \multirow[b]{2}{*}{ Autores } & \multicolumn{4}{|c|}{ Característica de la muestra } & \multicolumn{2}{|l|}{ Tratamiento } & \multicolumn{2}{|l|}{ Resultados } \\
\hline & País & $\begin{array}{c}\text { Diagnóstico } \\
\text { droga consumida }\end{array}$ & Sexo & Tamaño & Sesiones & Herramientas & Consumo de sustancias & POMFR \\
\hline \multirow[t]{3}{*}{$\begin{array}{l}\text { Black and } \\
\text { Amaro, } \\
2019\end{array}$} & \multirow[t]{3}{*}{ USA } & \multirow[t]{3}{*}{$\begin{array}{c}\text { SUD / } \\
52.5 \% \text { Cannabis, } \\
50 \% \text { Alcohol } \\
\text { y } 13 \% \text { cocaína }\end{array}$} & \multirow[t]{3}{*}{$\begin{array}{c}3100 \% \text { mujeres } \\
0 \% \text { hombres }\end{array}$} & \multirow[t]{3}{*}{225} & $\begin{array}{l}\text { Todos los participantes } \\
\text { recibieron la mismas } \\
\text { intervención TAU. }\end{array}$ & \multirow[t]{3}{*}{ PACS } & \multirow{3}{*}{$\begin{array}{l}\text { El tiempo tuvo un efecto significativo } \\
\text { en ambos grupos en la reducción } \\
\text { del craving; El grupo experimental } \\
\text { mostró una diferencia pequeña } \\
\text { respecto a los síntomas de craving } \\
(\mathrm{d}=0.28) \text { en comparación con la } \\
\text { intervención de NA. }\end{array}$} & \multirow[t]{3}{*}{30} \\
\hline & & & & & $\begin{array}{l}\text { MMWR: } 2 \text { sesiones de } \\
80 \text { minutos por semana, } \\
\text { durante } 6 \text { semanas. }\end{array}$ & & & \\
\hline & & & & & $\begin{array}{l}\text { NA: } 2 \text { sesiones de } 80 \text { minutos } \\
\text { por semana, durante } 6 \\
\text { semanas. }\end{array}$ & & & \\
\hline $\begin{array}{c}\text { Price } \\
\text { et al., } 2019\end{array}$ & USA & $\begin{array}{c}\text { SUD } \\
\text { 39\% Alcohol. } \\
\text { 24\% Marihuana }\end{array}$ & $\begin{array}{c}100 \% \text { mujeres } \\
0 \% \text { hombres }\end{array}$ & 187 & $\begin{array}{l}\text { TAU: } 2 \text { o } 3 \text { sesiones grupales } \\
\text { de } 1.5 \text { horas, durante } 10 \\
\text { a } 14 \text { semanas, incluyendo } \\
\text { el mínimo de } 1 \text { sesión } \\
\text { individual por mes. } \\
\text { MABT: } 8 \text { sesiones } \\
\text { individuales de } 1.5 \text { horas, } \\
\text { durante } 10 \text { semanas. } \\
\text { WHE: } 8 \text { sesiones individuales } \\
\text { de } 1.5 \text { horas, durante } 10 \\
\text { semanas. }\end{array}$ & $\begin{array}{l}\text { TLFB; } \\
\text { PACS }\end{array}$ & $\begin{array}{l}\text { Las intervenciones de MABT y WHE } \\
\text { generaron más días de abstinencia, a } \\
\text { los } 6 \text { meses (mean difference = 11.6); } \\
\text { mientras que a los } 12 \text { meses MABT } \\
\text { generó más días de abstinencia que } \\
\text { TAU y WHE (mean difference = 22.4; } \\
\text { d = 0.32). Respecto con el craving, el } \\
\text { grupo MBAT obtuvo una reducción } \\
\text { significativa en comparación con } \\
\text { el grupo TAU a los } 3 \text { meses (mean } \\
\text { difference }=-3.2 \text { ) a los } 6 \text { meses } \\
\text { (mean difference }=5.5 \text { ) y a los } 12 \\
\text { meses (mean difference }=-4.0 \text { ). }\end{array}$ & 22 \\
\hline
\end{tabular}




\begin{tabular}{|c|c|c|c|c|c|c|c|c|}
\hline \multirow[b]{2}{*}{ Autores } & \multicolumn{4}{|c|}{ Característica de la muestra } & \multicolumn{2}{|l|}{ Tratamiento } & \multicolumn{2}{|l|}{ Resultados } \\
\hline & País & $\begin{array}{c}\text { Diagnóstico } \\
\text { droga consumida }\end{array}$ & Sexo & Tamaño & Sesiones & Herramientas & Consumo de sustancias & POMFR \\
\hline \multirow[t]{2}{*}{$\begin{array}{l}\text { Witkiewitz } \\
\text { et al., } 2019\end{array}$} & \multirow[t]{2}{*}{ USA } & \multirow[t]{2}{*}{ AUD } & \multirow[t]{2}{*}{$\begin{array}{l}40.5 \% \text { mujeres } \\
59.5 \% \text { hombres }\end{array}$} & \multirow[t]{2}{*}{84} & $\begin{array}{l}\text { MBRP + tDCS activo: } 30 \text { minutos } \\
\text { de tDCS ( } 2.0 \text { miliamperios) y } \\
\text { meditación, más } 90 \text { minutos } \\
\text { de MBRP grupal, una vez a la } \\
\text { semana, durante } 8 \text { semanas. }\end{array}$ & $\begin{array}{c}\text { TLFB; } \\
\text { PACS: } \\
\text { observación }\end{array}$ & \multirow{2}{*}{$\begin{array}{l}\text { Los resultados muestran una } \\
\text { reducción significativa de las } \\
\text { bebidas consumidas por día a lo } \\
\text { largo del tiempo }\left(B=-0.53^{\star * \star}\right) \text {, } \\
\text { también respecto al número de } \\
\text { terapia atendidas }\left(B=-0.259^{\star *}\right) \text {. }\end{array}$} & \multirow[t]{2}{*}{27} \\
\hline & & & & & $\begin{array}{l}\text { MBRP + tDCS impostora: } \\
30 \text { minutos de tDCS }(0 \\
\text { miliamperios) y meditación, } \\
\text { más } 90 \text { minutos de MBRP } \\
\text { grupal, una vez a la semana, } \\
\text { durante } 8 \text { semanas. }\end{array}$ & & & \\
\hline \multirow[t]{2}{*}{$\begin{array}{l}\text { Zgierska } \\
\text { et al., } 2019\end{array}$} & \multirow[t]{2}{*}{ USA } & \multirow[t]{2}{*}{ AUD } & \multirow[t]{2}{*}{$\begin{array}{l}43.8 \% \text { mujeres } \\
56.2 \% \text { hombres }\end{array}$} & \multirow[t]{2}{*}{123} & $\begin{array}{l}\text { TAU: } \quad \text { intervenciones } \\
\text { ambulatorias basadas en } 12 \\
\text { pasos, CBT y autoayuda. }\end{array}$ & \multirow[t]{2}{*}{$\begin{array}{l}\text { Timeline; } \\
\text { Método de } \\
\text { regresión; DIC }\end{array}$} & $\begin{array}{l}\text { Al finalizar el tratamiento, el } \\
29.8 \% \text { del grupo MBRP-A y } \\
\text { el } 27.3 \% \text { de TAU reportaron }\end{array}$ & \multirow[t]{2}{*}{27} \\
\hline & & & & & $\begin{array}{l}\text { 2. TAU + MBRP-A: TAU + } \\
\text { sesiones semanales de } 2 \text { horas, } \\
\text { a lo largo de } 8 \text { horas. }\end{array}$ & & $\begin{array}{l}\text { “ningún consumo"; en el } \\
\text { seguimiento a las } 18 \text { semanas, el } \\
28.5 \% \text { del grupo MBRP-A reportó } \\
\text { ningún consumo; no existieron } \\
\text { diferencias entre los grupos. } \\
\text { Respecto a los días atendidos, en } \\
\text { el grupo MBRP-A, los resultados } \\
\text { muestran que los participantes } \\
\text { que asistieron a } 4 \text { o más sesiones } \\
\left(r=-0.34^{\star}\right) \text { y que practicaron en } \\
\text { casa }\left(r=-0.304{ }^{\star}\right) \text { disminuyeron } \\
\text { las bebidas consumidas por día } \\
\text { al finalizar el tratamiento. }\end{array}$ & \\
\hline
\end{tabular}




\begin{tabular}{|c|c|c|c|c|c|c|c|c|}
\hline \multirow[b]{2}{*}{ Autores } & \multicolumn{4}{|c|}{ Característica de la muestra } & \multicolumn{2}{|l|}{ Tratamiento } & \multicolumn{2}{|l|}{ Resultados } \\
\hline & País & $\begin{array}{c}\text { Diagnóstico } \\
\text { droga consumida }\end{array}$ & Sexo & Tamaño & Sesiones & Herramientas & Consumo de sustancias & POMFR \\
\hline \multirow[t]{2}{*}{$\begin{array}{l}\text { Brown } \\
\text { et al., } 2020\end{array}$} & \multirow[t]{2}{*}{ USA } & \multirow[t]{2}{*}{ AUD } & \multirow[t]{2}{*}{$\begin{array}{l}47.1 \% \text { mujeres } \\
52.9 \% \text { hombres }\end{array}$} & \multirow[t]{2}{*}{68} & $\begin{array}{l}\text { MBRP + tDCS activa: } 30 \text { minutos } \\
\text { de tDCS ( } 2.0 \text { miliamperios) y } \\
\text { meditación, más } 90 \text { minutos } \\
\text { de MBRP grupal, una vez a la } \\
\text { semana durante } 8 \text { semanas. }\end{array}$ & $\begin{array}{l}\text { Entrevista; } \\
\text { Concentración } \\
\text { de alcohol en } \\
\text { aliento. }\end{array}$ & \multirow{2}{*}{$\begin{array}{l}\text { Craving }\left(B=-0.28^{\star}\right) \text { y amplitudes } \\
\text { LPP }\left(B=-0.28^{* * \star}\right) \text { tuvieron un } \\
\text { decremento significativo a lo largo } \\
\text { del tiempo; pero al comparar } \\
\text { MBRP + tDCS activo y MBRP + tDCS } \\
\text { impostor no existieron diferencias } \\
\text { significativas; mientras que } \\
\text { atender a más sesiones de MBRP } \\
\text { se asoció con el decremento en el } \\
\text { nivel de craving }\left(B=0.28^{\star}\right) \text {. }\end{array}$} & \multirow[t]{2}{*}{27} \\
\hline & & & & & $\begin{array}{l}\text { MBRP + tDCS impostora: } \\
30 \text { minutos de tDCS }(0 \\
\text { miliamperios) y meditación, } \\
\text { después, más } 90 \text { minutos de } \\
\text { MBRP grupal, una vez a la } \\
\text { semana a lo largo de } 8 \text { semanas. }\end{array}$ & & & \\
\hline \multirow[t]{2}{*}{$\begin{array}{l}\text { Machado } \\
\text { et al., } 2020\end{array}$} & \multirow[t]{2}{*}{ Brasil } & \multirow[t]{2}{*}{$\begin{array}{c}\text { SUD / Alcohol, } \\
\text { cocaína, y cannabis. }\end{array}$} & \multirow[t]{2}{*}{$\begin{array}{l}50 \% \text { mujeres } \\
50 \% \text { hombres }\end{array}$} & \multirow[t]{2}{*}{42} & $\begin{array}{l}\text { TAU: incluyó tratamiento } \\
\text { psicológico, psiquiátrico y } \\
\text { nutricional. }\end{array}$ & $\begin{array}{l}\text { Regresión en } \\
\text { el tiempo. }\end{array}$ & \multirow{2}{*}{$\begin{array}{l}\text { Acerca del comportamiento } \\
\text { de consumo, los resultados no } \\
\text { refieren cambios estadísticos. } \\
\text { Pese a ello, el consumo de alcohol } \\
\text { en el grupo experimental fue de } \\
=1.5 \text { (pre tratamiento) a } \mathrm{m}=1.3 \\
\text { (seguimiento), el consumo de } \\
\text { marihuana de } \mathrm{m}=0.7 \text { a } \mathrm{m}=0.4 \mathrm{y} \\
\text { los días que se usaba cocaína de } \mathrm{m} \\
=9.3 \text { a } \mathrm{m}=4.5 \text {. }\end{array}$} & \multirow[t]{2}{*}{16} \\
\hline & & & & & $\begin{array}{l}\text { MBRP + TAU: TAU + } 2 \text { sesiones } \\
\text { semanales de psicoeducación y } \\
\text { mindfulness, } 8 \text { en total. }\end{array}$ & & & \\
\hline
\end{tabular}

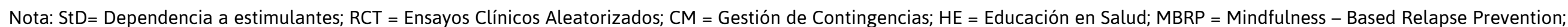

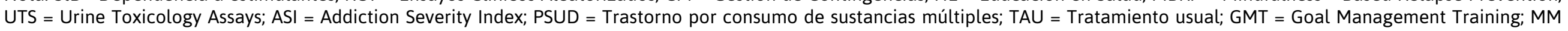

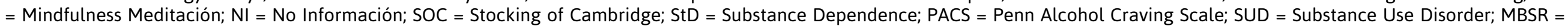

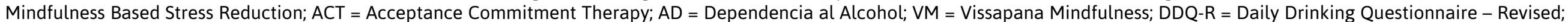

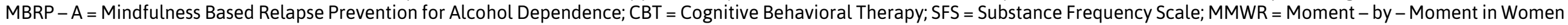

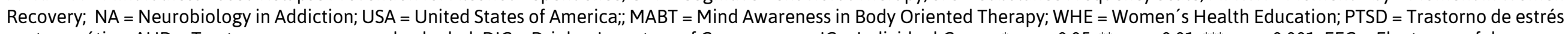

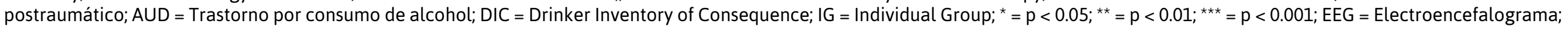
$y$, LPP $=$ Late Positive. 


\section{Discusión}

La presente revisión sistemática tuvo por objetivo general presentar la evidencia disponible sobre la eficacia que muestran las $\mathrm{MBI}$ en el tratamiento de las adicciones al alcohol, el cannabis y la cocaína. En respuesta, se identificó que en siete investigaciones se reportó que las $\mathrm{MBI}$ son eficaces para reducir el deseo de consumo al finalizar el tratamiento; sin embargo, pese a que Brown et al. (2019) indica que esta condición previene las recaídas en el consumo, apenas en una investigación se reportó que la reducción del craving se mantuvo en el seguimiento de hasta 12 meses (Price et al., 2019).

Por otra parte, respecto al consumo de alcohol, se reportó una remisión de la conducta adictiva al finalizar el tratamiento (Wongtongkam et al., 2017), en el seguimiento a los dos meses (Witkiewitz et al., 2019), a los seis meses (Davis et al., 2018) y a los 12 meses (Price et al., 2019). Mientras que, sobre el consumo de cocaína, Glasner et al. (2016), señalaron que las MBI muestran ser eficaces para reducir la ingesta de esta sustancia cuando, de manera concomitante a la adicción, el paciente presenta ansiedad o depresión. Finalmente, en relación con el consumo de cannabis, Machado et al. (2020), refirieron que los pacientes que recibieron MABT redujeron la cantidad de marihuana consumida, en adición, Price et al. (2019) identificaron que la abstinencia se mantuvo hasta el seguimiento a los 12 meses.

En este sentido, Witkiewitz et al. (2019) y Zgierska et al. (2019) encontraron una correlación inversamente proporcional entre la asistencia a sesiones de $\mathrm{MBI}$, cantidad de tiempo que se practica Mindfulness y consumo de sustancias, el incremento en las dos primeras reduciría la tercera. Al respecto, en tres investigaciones se identificó que la tasa de asistencia y permanencia fue mayor en las MBI que en las intervenciones que se ofrecieron al grupo control (Zgierska et al., 2017; Shorey et al., 2017; y Witkiewitz et al., 2019), además, Glasner et al. (2016) y Price et al. (2019) reportaron, en el seguimiento posterior, un incremento de la habilidad para practicar mindfulness entre los participantes del grupo experimental.

Es importante señalar que la $\mathrm{MBI}$ empleada con mayor frecuencia dentro de los estudios fue la MBRP, en su implementación, se identificaron las siguientes características comunes: fue un proceso de tratamiento grupal en el que se abordaron ejercicios de meditación guiada y de prevención de recaídas, incluyó tareas para casa; y, se aplicó en ocho sesiones, mismas que oscilaron entre los 75 y 120 minutos (Brown et al., 2020; Davis et al., 2018; Glasner et al., 2016; Wikiewitz et al., 2019; Zgierska et al., 2017; Zgierska et al., 2019; y, Zemestani y Ottaviani, 2016).

\section{Limitaciones}

Dentro de las limitaciones, se puede mencionar tres. La primera, en relación con la muestra, a excepción de Davis et al. (2018), Witkiewitz et al. (2019) y Zgierska et al. (2019), ninguna de las investigaciones reportó o alcanzó el número mínimo de participantes para realizar mediciones fiables y arrojar datos con un efecto medio; por cuanto, se 
limita la posibilidad de que los puedan ser generalizados a otras poblaciones.

Por otra parte, la segunda limitación se relaciona con el seguimiento. De acuerdo con Bowen et al. (2014), las MBI procuran modificaciones significativas en la conducta adictiva hasta los 12 meses después de haber finalizado el tratamiento. Dentro de los artículos revisados, a excepción de las investigaciones desarrolladas por Davis et al. (2018), Price et al. (2019) y Zgierska et al. (2017), el tiempo de seguimiento fue menor a cinco meses, de hecho, en dos estudios no se realizó seguimiento (Shorey et al., 2017 y Valls et al., 2016).

Esta situación limitó, por una parte, la posibilidad de observar los cambios que se producen en la conducta adictiva con el tiempo y la práctica, que Witkiewitz et al. (2019) y Zgierska et al. (2019) señalan se correlacionan de manera inversamente proporcional; y, por otra, la de medir si los cambios alcanzados durante el tratamiento se mantuvieron a lo largo del tiempo y como estos influyeron sobre el deseo y la remisión del consumo.

Finalmente, la tercera limitación se relaciona con la cantidad de ECA en los que se abordan intervenciones sobre adicción al cannabis y a la cocaína. Las drogas ilegales que se consumen con mayor frecuencia a nivel mundial son la marihuana y la cocaína (19 (UNOCD, 2019 y Organización de Estados Americanos, 2019). Sin embargo, apenas cuatro investigaciones abordaron el consumo de marihuana (Machado et al., 2020 y Price et al., 2019) y el de cocaína (Glasner et al., 2016 y Machado et al., 2020); lo que acentúa una brecha importante en el conocimiento sobre la eficacia que muestran las MBI para trabajar con estas psicopatologías.

\section{Futuros estudios}

En función de las limitaciones identificadas, se sugiere que en investigaciones futuras se empleen ECA en los que se compare las MBI con modelos de psicoterapia cuya implementación este sustentada en evidencia empírica, entre otras, Terapia Cognitivo Conductual, Terapia Conductual de Pareja, Terapia Sistémica y Entrenamiento Familiar (Lefio et al., 2013).

Debido a la existencia de múltiples $\mathrm{MBI}$, resultaría oportuno realizar investigaciones en las que se comparen dos o más de estos modelos de psicoterapia, con el objetivo de identificar la que mayor eficacia muestre. Además, es importante que en estos estudios se realicen análisis de poder para determinar el tamaño de la muestra, y de esta manera permitir la generalización de los resultados.

Por otra parte, se sugiere el incremento de investigaciones sobre la implementación de $\mathrm{MBI}$ en el tratamiento de los Trastornos por consumo de Cannabis y de Cocaína, así como, el de múltiples sustancias; mismas que, contemplen la recogida de datos en el prey post tratamiento, al igual que, en el seguimiento de hasta mínimo 12 meses. De esta manera se conocería la eficacia que muestran las MBI para procurar la remisión continuada de la psicopatología. 


\section{Implicaciones Teóricas}

De acuerdo con lo señalado por Chiesa y Serreti (2014), en su revisión sistemática, el componente de las MBI que trabajaría sobre la prevención de recaídas y el craving es el incremento de habilidades de mindfulness. En este sentido, en la presente revisión sistemática se identificó que a mayor práctica de mindfulness incrementan también los días en abstinencia y disminuye el deseo de consumo.

Esto podría explicarse, y permite reforzar, el planteamiento teórico, de que las MBI funcionan a través del mecanismo bottom up (Korecki et al., 2020). Es decir, se experimenta el deseo de consumo, pero, no se juzga como positivo o negativo, se lo acepta; lo que, a la larga permitirá una habituación a las sensaciones y disminuirá la posibilidad de recaer; pues, no existe intención alguna de impedir la experiencia del sentir la emoción.

\section{Implicaciones Clínicas}

Para efectos prácticos, de acuerdo con lo señalado por Korecki et al. (2020), se encontró que el entrenamiento en meditación, formal o informal, permite incrementar la capacidad para prestar atención al momento presente; $y$, se sitúa como el componente clave para prevenir recaídas, mientras mayor práctica menor probabilidad de recaer. De ahí que, la eficacia de las $M B I$ esté determinada por su capacidad para promover la adherencia de los pacientes al tratamiento, mismo que, se sugiere sea de ocho sesiones, en formato grupal, con un tiempo de duración de entre 75 y 120 minutos; además, resulta oportuno establecer sesiones de seguimiento que puedan extenderse hasta los 12 meses.

Por otra parte, contrario a las revisiones realizadas por Korecki et al. (2020), y Chiesa y Serreti (2014), que se centraron en MBI para los TCS, sin especificar una sustancia; la presente revisión sistemática se centró en tres sustancias (alcohol, cannabis y cocaína), encontrando que la MBRP, en el formato sugerido por Bowen et al. (2011), es el modelo terapéutico que se emplea con mayor frecuencia para intervenir sobre estos trastornos, además, existe la MBRP-A para intervenir en específico sobre el TCA.

Finalmente,enconcordanciaconloexpuesto por Chiesa y Serreti (2014), los resultados mencionados con antelación permiten señalar que las MBI son importantes para momentos posteriores en las intervenciones, en las que el objetivo es trabajar sobre prevención de recaídas; además, muestran eficacia para aplicarse cuando, a parte de la adicción, existe un trastorno comórbido (ansiedad o depresión).

\section{CONCLUSIÓN}

Las MBI muestran eficacia para, a partir del entrenamiento en meditación, manejar el craving, prevenir recaídas, reducir la intensidad y frecuencia del consumo de sustancias; así como, para regular el estado de ánimo, lo que las convierte en modelos psicoterapéuticos útiles para abordar las TCS; en especial, cuando se las utiliza en momentos avanzados de la intervención y cuando coexisten, junto con la adicción, trastornos de ansiedad o depresión. 
Sin embargo, los resultados expuestos en la presente revisión sistemática no son concluyentes, tampoco generalizables, pues los artículos incluidos son heterogéneos en cuanto a su calidad metodológica. Pese a ello, se encontró que la información expuesta permite reforzar el planteamiento teórico en el que se sugiere que las $\mathrm{MBI}$ funcionan a través del modelo bottom up; así como, que el éxito de estas intervenciones está determinado por la práctica constante.

\section{Rol de los fondos o recursos}

No existió una fuente de financiamiento para el desarrollo del artículo.

\section{Conflicto de interés}

Los autores declaran que no ha recibido ninguna contribución económica, o mantiene una relación que pueda suponer un conflicto de interés respecto al contenido presentado.

\section{Agradecimientos}

Los autores agradecen a la Dra. Roser Granero de la Universidad Autónoma de

Barcelona, miembro del grupo PAD; así como, a Smart UniverCity y Postgrados Universidad Católica de Cuenca por su constante e invaluable apoyo

\section{REFERENCIAS}

Asociación Americana de Psiquiatría. (2014). Manual diagnóstico y estadístico de los Trastornos Mentales versión V. Editorial Panamericana

Arnold, P., y Bautmans, I. (2014). The influence of strength training on muscle activation in elderly persons: A systematic review and meta-analysis. Experimental Gerontology, 58(1), 58-68. https://doi.org/10.1016/j. exger.2014.07.012

Becoña, E., y Cortés, M. (2010). Manual de adicciones para psicólogos especialistas en psicología clínica en formación. Editorial Sociodrogalcohol.

Bautista, T., James, D., y Amaro, H. (2019). Acceptability of mindfulness-based interventions for substance use disorder: A systematic review. Complementary Therapies in Clinical Practice, 35(1), 201-207. https://pubmed.ncbi.nlm.nih. gov/31003659/

Bayir, B., y Aylaz, R. (2020). The effect of mindfulness-based education given to individuals with substance-use disorder according to self-efficacy theory on selfefficacy perception. Applied Nursing Research, 57(1), 1-9. https://pubmed.ncbi. nlm.nih.gov/32907766/

Black, D. S. (2014). Mindfulness-based interventions: An antidote to suffering in the context of substance use, misuse, and addiction. Substance Use and Misuse, 49(5), 487-491. https://doi.org/10.3109/10826084. 2014.860749

Black, D. S., y Amaro, H. (2019). Moment-byMoment in Women's Recovery (MMWR): Mindfulness-based intervention effects on residential substance use disorder treatment retention in a randomized controlled trial. Behaviour Research and Therapy, 120(2019), 1-11. https://doi. org/10.1016/j.brat.2019.103437

Bowen, S., Witkiewitz, K., Chawla, N., y Grow, J. (2011). Integrating mindfulness meditation and cognitive behavioral traditions for the long-term treatment of addictive behaviors. Journal of Clinical Outcomes Management, 18(10), 473-479. https://www.eomega.org/ sites/default/files/resources/sm14-2605898.requiredreading.pdf 
Bowen, S., Witkiewitz, K., Clifasefi, S., Grow, J., Chawla, N., Hsu, S., Carroll, H., Harrop, E., Collins, S., Lustyk, M., y Larimer, M. (2014). Relative efficacy of mindfulness-based relapse prevention, standard relapse prevention, and treatment as usual for substance use disorders. JAMA Psychiatry, 71(5), 547-556. https://pubmed.ncbi.nlm. nih.gov/24647726/

Brewer, J. A., Bowen, S., Smith, J. T., Marlatt, G. A., y Potenza, M. N. (2010). Mindfulness-based treatments for co-occurring depression and substance use disorders: What can we learn from the brain?.Addiction, 105(10), 1698-1706. https://doi.org/10.1111/j.13600443.2009.02890.x

Brown, D. R., Jackson, C. T., Claus, E. D., Votaw, V. R., Stein, E. R., Robinson, S. H., Wilson, A. D., Brandt, E., Fratzke, V., Clark, V. P., y Witkiewitz, K. (2020). Decreases in the Late Positive Potential to Alcohol Images Among Alcohol Treatment Seekers Following Mindfulness-Based Relapse Prevention. Alcohol and Alcoholism, 55(1), 78-58. https://doi.org/10.1093/alcalc/agz096

Cavicchioli, M., Movalli, M., y Maffei, C. (2018). The Clinical Efficacy of MindfulnessBased Treatments for Alcohol and Drugs Use Disorders: A Meta-Analytic Review of Randomized and Nonrandomized Controlled Trials. European Addiction Research, 24(3), 137-162. https://doi. org/10.1159/000490762

Centro Cochrane Iberoamericano. (2011). Manual Cochrane de Revisiones Sistemáticas de Intervenciones, versión 5.1. 0. The Cochrane Collaboration. https:// n9.cl/Ogabw

Ciapponi, A. (2018). AMSTAR-2: herramienta de evaluación crítica de revisiones sistemáticas de estudios de intervenciones de salud. Evidencia, Actualizacion en la Práctica Ambulatoria, 21(1), 4-13. https:// doi.org/10.51987/evidencia.v21i1.6834

Chiesa, A., y Serretti, A. (2014). Are MindfulnessBased Interventions Effective for Substance
Use Disorders? A Systematic Review of the Evidence. Substance Use y Misuse, 49(1), 492-512. https://doi.org/10.3109/10826084. 2013.770027

Davis, J. P., Berry, D., Dumas, T. M., Ritter, E., Smith, D. C., Menard, C., y Roberts, B. W. (2018). Substance use outcomes for mindfulness based relapse prevention are partially mediated by reductions in stress: Results from a randomized trial. Journal of Substance Abuse Treatment, 91(1), 37-48. https://doi.org/10.1016/j.jsat.2018.05.002

Grant, S., Colaiaco, B., Motala, A., Shanman, R., Booth, M., Sorbero, M., y Hempel, S. (2017). Mindfulness-based Relapse Prevention for Substance Use Disorders: A Systematic Review and Meta-analysis. Journal of Addiction Medicine, 11(5), 386-396. https:// doi.org/10.1097/ADM.0000000000000338

Glasner, S., Mooney, L. J., Ang, A., Chokron, H., Hartwell, E., Brecht, M., y Rawson, R. R. (2016). Mindfulness-Based Relapse Prevention for Stimulant Dependent Adults: A Pilot Randomized Clinical Trial. Mindfulness (NY), 8(1), 126-135. https://pubmed.ncbi. nlm.nih.gov/28191264/

Hervás, G., Cebolla, A., y Soler, J. (2016). Intervenciones psicológicas basadas en mindfulness y sus beneficios: estado actual de la cuestión. Clínica y Salud, 27(3), 115-124. https://doi.org/10.1016/j.clysa.2016.09.002

Korecki, J. R., Schwebel, F. J., Votaw, V. R., y Witkiewitz, K. (2020). Mindfulness-based programs for substance use disorders: a systematic review of manualized treatments. Substance Abuse: Treatment, Prevention, and Policy, 15(1), 1-37. https:// doi.org/10.1186/s13011-020-00293-3

Lefio, L. A., Villarroel, S. R., Rebolledo, C., Zamorano, P., y Rivas, K. (2013). Intervenciones eficaces en consumo problemático de alcohol y otras drogas. Revista Panamericana de Salud Publica, 34(4), 257-266. https://iris.paho.org/ bitstream/handle/10665.2/8977/07 .pdf? sequence $=1$ ctisAllowed $=y$ 
Lerma, J. M., Steinebach, P., Carvajal, F., Ulloa, V., Cid-Parra, C., y Langer, Á. I. (2015). Risk factors associated with problematic alcohol use in adolescence: The preventive role of mindfulness. Psychology, Society and Education, 7(1), 57-69. https://doi. org/10.25115/psye.v7i1.540

Li, W., Howard, M. O., Garland, E. L., McGovern, P., y Lazar, M. (2017). Mindfulness treatment for substance misuse: A systematic review and meta-analysis. Journal of Substance Abuse Treatment, 75(1). https://doi. org/10.1016/j.jsat.2017.01.008

Machado, M. P., Fidalgo, T. M., Brasiliano, S., Hochgraf, P. B., y Noto, A. R. (2020). The contribution of mindfulness to outpatient substance use disorder treatment in Brazil: a preliminary study. Brazilian Jounal of Psychiatry, 00(00), 1-5. https://doi. org/10.1590/1516-4446-2019-0725

McLellan, A. T., Lewis, D. C., O’Brien, C. P., y Kleber, H. D. (2000). Drug dependence, a chronic medical illness implications for treatment, insurance, and outcomes evaluation. Journal of the American Medical Association, 284(13), 1689-1695. https://doi. org/10.1001/jama.284.13.1689

Moher, D., Liberati, A., Tetzlaff, J., y Altman, D. G., (2009). Preferred reporting items for systematic reviews and meta-analyses: The PRISMA statement. PLoS Medicine, 6(7). 1-8. https://doi.org/10.1371/journal. pmed.1000097

Oficina de las Naciones Unidas contra la Droga y el Delito. (2019). Informe Mundial sobre las Drogas 2019. https://wdr.unodc.org/ wdr2019/prelaunch/WDR2019_B1_S.pdf

Organización Panamericana de la Salud.(2003). Clasificación Estadística Internacional de Enfermedades y Problemas Relacionados con la Salud. Decima Revisión. Editorial Oganización Panamericana de la Salud. https://iris.paho.org/handle/10665.2/6283

Organización de Estados Americanos. (2019). Informe sobre le consumo de drogas en Las
Américas 2019. Organización de Estados Americanos. https://n9.cl/44m29

Öst, L. G. (2008). Efficacy of the third wave of behavioral therapies: A systematic review and meta-analysis. Behaviour Research and Therapy, 46(3), 296-321. https://doi. org/10.1016/j.brat.2007.12.005

Price, C. J., Adams, E. Croell, S., y Pike, K. (2019). Longitudinal effects of interoceptive awareness training through mindful awareness in body-oriented therapy (MABT) as an adjunct to women's substance use disorder treatment: A randomized controlled trial. Drugs and Alcohol Dependence, 198(1), 140-149. https://doi. org/10.1016/j.drugalcdep.2019.02.012

Sancho, M., De Gracia, M., Rodríguez, R. C., Mallorquí-Bagué, N., Sánchez-González, J., Trujols, J., Sánchez, I., Jiménez-Murcia, S., y Menchón, J. M. (2018). Mindfulness-based interventions for the treatment of substance and behavioral addictions: A systematic review. Frontiers in Psychiatry, 9(1), 1-9. https://doi.org/10.3389/fpsyt.2018.00095

Sateler, A., Pino, G., López, A., Silva, L., Solari, S., Duffau, B., y Ríos, J. C. (2019). Popular names and classification of illicit drugs commonly used in Chile. Revista Medica de Chile, 147(12), 1613-19620. https://doi. org/10.4067/S0034-98872019001201613

Shorey, R. C., Elmquist, J., Gawrysiak, M. J., Strauss, C., Haynes, E., Anderson, S., y Stuart, G. L. (2017). A Randomized Controlled Trial of a Mindfulness and Acceptance Group Therapy for Residential Substance Use Patients. Substance Use \& Misuse, 52(11), 1400-1410. https://doi.org/ 10.1080/10826084.2017.1284232

Skanavi, S., Laqueille, X., y Aubin, H. J. (2011). Interventions basées sur la pleine conscience en addictologie. Encephale, 37(5), 379-387. https://doi.org/10.1016/j. encep.2010.08.010

Sloan, E., Hall, K., Moulding, R., Bryce, S., Mildred, $\mathrm{H}$ y Staiger, P. K. (2017). Emotion regulation 
as a transdiagnostic treatment construct across anxiety, depression, substance, eating and borderline personality disorders: A systematic review. Clinical Psychology Review, 57(1), 141-163. https://doi. org/10.1016/j.cpr.2017.09.002

Swain, J., Hancock, K., Hainsworth, C., y Bowman, J. (2013). Acceptance and commitment therapy in the treatment of anxiety: A systematic review. Clinical Psychology Review, 33(8), 965-978. http:// dx.doi.org/10.1016/j.cpr.2013.07.002.

Ursa, A. (2018). La meditación como práctica preventiva y curativa en el sistema nacional de salud. Medicina Naturista, 12(1), 4753. https://dialnet.unirioja.es/servlet/ articulo?codigo $=6267068$

Valls, C., Caracuel, A., y Verdejo, A. (2016). Goal Management Training + Mindfulness Meditation improves executive functions and transfers to ecological tasks of daily life in polysubstance users enrolled in therapeutic community treatment. Drug and Alcohol Dependence, 165(1), 9-14. http:// doi.org/10.1016/j.drugalcdep.2016.04.040.

Witkiewitz, K., y Villarroel, N. A. (2009). Dynamic Association Between Negative Affect and Alcohol Lapses Following Alcohol Treatment. Journal of Consulting and Clinical Psychology, 77(4), 633-644. https:// doi.org/10.1037/a0015647

Witkiewitz, K., Bowen, S., Douglas, H., y Hsu, S. (2013). Mindfulness-based relapse prevention for substance craving. Addictive Behaviors, 38(2), 1563-1571. https://doi. org/10.1016/j.addbeh.2012.04.001

Witkiewitz, K., Stein, E., Votaw, V., Wilson, A., Roos, C., Gallegos, S., Clark, V., y Claus, E, D. (2019). Mindfulness-Based Relapse Prevention and Transcranial Direct Current Stimulation to Reduce Heavy Drinking: A Double-Blind Sham-Controlled Randomized Trial. Alcoholsm Clinical and Experimental Research, 43(6), 1296-1307. https://doi.org/10.1111/acer.14053
Wongtongkam, N., Lampoo, S., Choocherd, P., y Chiangkuntod, S. (2017). Partial Efficacy of Vipassana Mindfulness Approach in Alcohol-Dependent Persons. Alcoholism Treatment Quartely, 36(1), 3-14. https://doi. org/10.1080/07347324.2017.1355224

Zemestani, M., y Ottaviani, C. (2016). Effectiveness of Mindfulness-Based Relapse Prevention for Co-occurring Substance Use and Depression Disorders. Mindfulness, 7(1), 1347-1355. https://link.springer.com/ article/10.1007\%2Fs12671-016-0576-y

Zgierska, A., Rabago, D., Chawla, N., Kushner, K., Koehler, R., y Marlatt, A. (2009). Mindfulness meditation for substance use disorders: A systematic review. Substance Abuse, 30(4), 266-294. https://doi. org/10.1080/08897070903250019

Zgierska, A., Shapiro, J., Burzinski, C., Lerner, F., y Goodman, V. (2017). Maintaining Treatment Fidelity of Mindfulness-Based Relapse Prevention Intervention for Alcohol Dependence: A Randomized Controlled Trial Experience. EvidenceBased Complementary and Alternative Medicine, 2017, 1-13. https://doi. org/10.1155/2017/9716586

Zgierska, A., Burzinski, C., Mundt, M., McClintock, A., Cox, J., Coe, C., Miller, M., y Fleming, M. (2019). Mindfulness-based relapse prevention for alcohol dependence: Findings from a randomized controlled trial. Journal of Substance Abuse Treatment, 100(1), 8-17. https://doi.org/10.1016/j. jsat.2019.01.013 